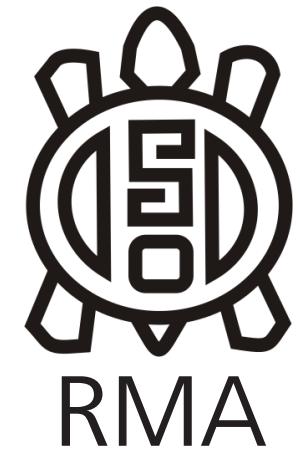

Antropología Social

\title{
Entre abrazos y el dolor. Un análisis etnográfico del reclamo público a partir de una muerte en Capilla del Monte (Córdoba)
}

\author{
Between hugs and pain. An ethnographic analysis of the public \\ reclamation from a death in Capilla del Monte (Córdoba)
}

Agustín Villarreal*

*Instituto de Antropología de Córdoba (IDACOR - CONICET); E-mail: agus.villarreal1901@gmail.com

\begin{abstract}
Resumen
En este trabajo propongo abordar las expresiones públicas del reclamo por la muerte de un joven de 17 años en la comisaría de Capilla del Monte (Córdoba- Argentina). Esto produjo la conformación de un grupo asambleario para reclamar por el joven y la violencia institucional, lo que provocó diversas movilizaciones en la ciudad y en algunos de ellos participó la familia.

En este marco, mi interés se constituye en un análisis procesual sobre los aniversarios de la muerte para problematizar el lugar de la figura familiar. Esto me permite comprender los sentidos locales sobre disputas y encuentros que se constituyeron en el reclamo por la violencia institucional al interior de la provincia de Córdoba. Las actividades políticas que se produjeron año a año logran socavar una problematización sobre la demanda de justicia y el rol de los familiares de víctimas en ellas.
\end{abstract}

Palabras clave: Muerte; Reclamo Público; Familia; Asamblea.

\begin{abstract}
In this paper I propose to address the public expressions of the claim made for the death of a 17-year-old boy in the police station of Capilla del Monte (Córdoba, Argentina). Such death led to the claim by a group of people for the dead of the young man and for institutional violence, resulting in several mobilizations in the city.

My paper focuses on a processual analysis of the anniversaries of the death of the young man to problematize the place of the family role. This has allowed me to understand the local meanings of disputes and encounters forged in the claim for institutional violence within the province of Córdoba. The political activities that took place year after year have undermined the problematization of the search for justice and the role of the victims' relatives in them.
\end{abstract}

Keywords: Death; Public Claim; Family; Assembly.

\section{Introducción}

En la ciudad de Capilla del Monte ${ }^{1}$ el 26 de octubre del 2013 fue detenido José ${ }^{2}$ con 17 años. Fue trasladado hasta la comisaría de la ciudad y luego de unas horas, su madre recibió el llamado del comisario que le explicaba que su hijo murió por un "suicidio". Sus padres que vivían en San Esteban ${ }^{3}$ se desplazaron hasta el lugar para entender qué había sucedido con el menor de edad.

\footnotetext{
1 Se encuentra en el departamento de Punilla, a 109 km de la ciudad capital cordobesa. Cuenta con alrededor de 9 mil habitantes y se caracteriza por ser una ciudad turística, sobre todo en épocas veraniegas. Se caracteriza por estar al pie del Cerro Uritorco, uno de los principales puntos turísticos de la provincia de Córdoba

2 Para el resguardo y la privacidad, se utilizarán nombres ficticios.

3 San Esteban es una comuna del departamento de Punilla que está a $7 \mathrm{Km}$ de Capilla del Monte. Tiene cerca de 700 habitantes y cuenta con una escuela primaria y un destacamento policial.
}

Mientras tanto, la noticia corrió entre los vecinos y amigos que también se fueron sumando y acercando hasta la puerta de la institución policial para acompañar a la familia y tratar de entender lo sucedido. Con el transcurso de las horas se fueron acercando más personas de ambas localidades luego de la difusión por redes sociales de lo sucedido al interior de la comisaría.

En un primer momento no le permitían el ingreso de la madre hasta la celda donde se hallaba el cuerpo de José, bajo la explicación de que los peritos estaban trabajando 4 . Sin embargo, a partir de la insistencia de los manifestantes que se apostaron en la puerta del lugar,

\footnotetext{
4 El uso de cursiva se utilizará durante este trabajo, para dar cuenta de enunciaciones que son emitidas por las personas con las que realicé el trabajo.
} 
dejaron ingresar a la familia y hablar con el comisario, que le comunicó que su hijo murió ahorcándose en el techo del calabozo en el que estaba detenido. La inhabilitación policial de dejarle ver el cuerpo de José a los padres, sumado al relato del suicidio que familiares y vecinos no creían, produjo una indignación general que devino en una pedrea en la puerta de la comisaría y la posterior represión por parte de la policía junto con gendarmería que se trasladó hasta la ciudad para custodiar la comisaría.

Esta muerte en la institución policial fue dirimida por familiares y vecinos como parte de las amenazas sufridas por el joven antes de morir. En una de las entrevistas que realicé con Alba -la madre- me explicó que en una de las últimas charlas que, tuvo con su hijo, le confesó que había agentes policiales de la ciudad que lo mandaban a robar y vender droga bajo amenaza.

Al día siguiente, ya sin la presencia de los padres, más vecinos se sumaron frente a la comisaría para plantear su rechazo a la versión del suicidio. Esto habilitó el reclamo del hostigamiento policial hacia los jóvenes en el departamento de Punilla. Hubo más de 200 personas reunidas y los medios de comunicación provincial se hicieron eco de la noticia de Capilla del Monte, dando cuenta que: "un joven había sido encontrado ahorcado dentro del calabozo" 5 .

Las fuerzas de seguridad volvieron a reprimir a los manifestantes allí apostados, mientras, seguían lloviendo piedras sobre la comisaría y otros edificios de la ciudad. Como consecuencia, los manifestantes se fueron reuniendo en la plaza principal en pequeños grupos, a dos calles de distancia del establecimiento policial. Allí acordaron seguir encontrándose para exigir el esclarecimiento de la muerte de José, construyendo la "Asamblea de Capilla del Monte", conformada por vecinos y familiares del joven y comenzaron a reunirse semanalmente en aquella plaza. Organizaron múltiples actividades y sumaron otros reclamos de violencia institucional de la zona ${ }^{6}$. En un primer momento la familia participó activamente de las reuniones, aunque luego surgieron disputas que los alejaron de la Asamblea.

Para este trabajo pretendo centrarme en los eventos de reclamo que se produjeron en los primeros tres años del aniversario de la muerte de José. A través de ellos, pretendo problematizar etnográficamente el activismo familiar, producto de un trabajo político atravesado por resistencias, disputas y contestaciones.

En consonancia con los eventos que voy a desandar

5 Fuente: www.lavoz.com.ar/sucesos/protesta-e-indignacion-encapilla-del-monte-por-un-joven-muerto-dentro-de-una-comisaria 6 Los miembros del grupo hacían referencia a la violencia institucional para dar cuenta de la violencia que ejercen las diversas instituciones estatales locales y provinciales, para no quedar reducido a la violencia policial. en este artículo, entre la familia y la Asamblea había acusaciones encontradas del uso político de José. Mientras que la madre acusaba al grupo asambleario de usar a su hijo para objetivos partidarios, ellos la acusaban a la abogada del caso de usar la muerte para la militancia de su agrupación.

Es por ello que procuro centrarme en las características que fueron adquiriendo las protestas con el transcurrir del tiempo, para comprender quiénes fueron parte del reclamo o no y cómo se produjeron esos eventos. Con la descripción etnográfica de estas situaciones sociales (Gluckman, 2003) pretendo describir y comprender los sentidos locales de reclamo de una muerte de violencia institucional al interior de la provincia de Córdoba.

\section{Al dolor lo llevamos nosotros. A un año de la muerte}

Seis meses antes de que se cumpliera el primer aniversario de la muerte de José, el grupo asambleario organizó una movilización hacia la fiscalía que tenía a cargo la causa judicial y para ello realizaron una reunión previa. En ese encuentro asistió Alba -la madre- con sus hijos y narró cuál era el estado de avance de la investigación. Sin embargo, luego se generó una discusión sobre la participación de banderas políticas ${ }^{7}$ para ese día, en la que ella estuvo al margen del debate y terminó retirándose antes con sus hijos.

Unas semanas posteriores a este encuentro ella decidió alejarse de la Asamblea y explicó que no quería que utilizaran a su hijo, a través de un comunicado que difundió por la red social Facebook. En uno de los encuentros que tuvimos me explicó que la decisión la había tomado luego de saber que hacían política para provechos partidarios. Por otro lado, la abogada le explicó que no era correcto realizar una marcha porque podría entorpecer la causa.

Esta situación generó distanciamiento entre la familia de José y la Asamblea. Al interior del grupo hubo miembros que también se alejaron y fueron pocos los que participaron de aquella concentración frente a la fiscalía judicial de la ciudad de Cosquín.

Luego de este evento en aquella ciudad, las reuniones en la plaza de Capilla del Monte de la Asamblea fueron cada vez más escasas. Sin embargo, querían realizar una manifestación pública para el 26 de octubre y rememorar el primer año de la muerte de José. Para ello, se produjo una reunión organizativa previa en el que se explicitó la necesidad de que el grupo familiar formara parte de la movilización pactada.

7 La discusión estaba referida sobre la presencia de las banderas políticas de las organizaciones que iban a participar en aquella jornada de movilización. Mientras algunos participantes de la reunión entendían que debían manifestarse sólo con la foto de José, otros hacían énfasis en la importancia de llevar las banderas de las organizaciones políticas a las que pertenecían como militantes. 
Valeria, era una de las asambleístas que mejor vínculo construyó con la familia y que participó en cada uno de los eventos, reuniones y movilizaciones que se organizaron desde la primera concentración frente a la comisaría. Fue quien enunció la importancia de que fuera Alba quien difundiera el evento en sus redes sociales como así también, escriba algo relacionado a la muerte de su hijo para que sea leído luego de la marcha.

Coincidieron en colocarse cintas negras en la parte del pecho del cuerpo y llevar fotos de José para que se repartan durante la manifestación y se las lleve en alto. La imagen elegida era la que Alba compartió días antes en las redes sociales junto a la frase "Justicia por José". Además, definieron, cuales iban a ser los puntos que debía tener la convocatoria para su posterior difusión, como Abajo el Código de Faltas ${ }^{8}$, Justicia por José y Juicio y Castigo para los responsables. En todas estas decisiones, sin embargo, no estuvo el posicionamiento de la familia, particularmente de Alba que decidió el distanciamiento asambleario.

Tanto las fotos en José en alto, como las cintas negras y la definición de los puntos de la convocatoria funcionaron como parte de la organización para el accionar ritualizado. Se trataron de medios para el objetivo ritual en el espacio público, a un año de la muerte de José cuyos significados estaban asociados a cargas emocionales (Turner, 1993).

Luego de la invitación realizada a la familia a participar del evento, Alba tenía miedo de asistir al acto en la plaza y que se vuelvan a producir incidentes con la policía. Sin embargo, a pesar de la molestia por el uso político de la muerte de José, decidió participar con dos de sus hijas.

Una vez que llegamos con la madre y sus hermanas a la plaza principal de Capilla del Monte, se fueron acercando diversas organizaciones políticas del departamento de Punilla, como la Jauretche ${ }^{9}$, Partido Obrero $^{10}$, la Mesa Departamental de Derechos Humanos de Punilla ${ }^{11}$, Papel secundario ${ }^{12}$ y el Partido

8 La Ley Contravencional n 8431 Código de Faltas de la Provincia de Córdoba ha sido denunciada públicamente por su aplicación. A partir de su inconstitucionalidad y la violación de Derechos Humanos que se producen, por las detenciones arbitrarias y discriminatorias que se ejercen sobre sectores específicos de la población. Desde el primero de abril de 2016 fue reemplazado por el Código de Convivencia, que sigue resultando inconstitucional y presenta ambigüedades, sobre términos vagos y pocos claro que le permite a la policía ejercer abuso de poder y violar derechos básicos de las personas, actuando de manera arbitraria.

9 Organización política ligado al kirchnerismo.

10 Partido político con orientación al trotskista.

11 La mesa de trabajo de Derechos Humanos es una organización vinculada a la Memoria, los Derechos Humanos y el Terrorismo de Estado. Como explica el registro de organizaciones sociales de la provincia de Córdoba, vinculados a este tema, tiene como objetivo contribuir al análisis de la realidad dentro del contexto local, departamental, provincial y nacional y recuperar la memoria histórica a través de las propias trayectorias de vida.

12 Son un grupo de estudiantes secundarios, algunos de ellos son militantes del Frente de Izquierda. Se manifiestan en defensa de la
Solidario ${ }^{13}$. Con estos partidos que tenían entre tres y cinco representantes cada uno, la mamá y sus dos hijas, y los miembros de la Asamblea, que eran alrededor de cinco personas, comenzó el acto.

Elvira y Mirta, eran dos señoras de la Asamblea. Mientras que Mirta que participaba activamente en las reuniones que realizaba el grupo, Elvira tenía una extensa trayectoria militante y dirigía una organización de Derechos Humanos del departamento de Punilla de Córdoba. Fue Mirta quién abrió el encuentro mientras estábamos en círculo dentro del espacio de la plaza que se llamaba el "Jardín de la Memoria"14. Comenzó agradeciendo la presencia de los participantes y pidió que la madre:

Yo vine acá a la plaza para pedir
justicia por José, ahora más que
nunca tengo fuerzas para seguir
adelante y luchar para que se haga
justicia. Él me da fuerzas para seguir
luchando. Para los que decían que
no iba a venir, acá estoy (Alba,
octubre 2014).

Para finalizar la ronda, expusieron sus discursos los representantes de los partidos que estaban presentes. Desde allí, Elvira propuso iniciar la marcha que se iba a desarrollar alrededor de la plaza, por pedido de ella, Alba encabezó la marcha.

Para finalizar el acto de conmemoración, Elvira pidió centrar las prácticas alrededor del cuerpo para que subiéramos los brazos en forma de agradecimiento y nos diéramos un abrazo fraternal para cerrar el evento. Una puesta en escena que construía una metáfora de agradecimiento y unión.

Con este primer aniversario se puso en escena los procesos políticos construidos en torno a la Asamblea como espacio de reclamo, mediante las acusaciones sobre el uso político que le daban. Previo a la movilización mientras estábamos esperando la llegada de más participantes, se suscitaron discusiones entre una de las hermanas de José y una asambleísta por las acusaciones en redes sociales de hacer política con la muerte de su familiar. Este mismo descontento manifestó la madre cuando nos encontramos previamente en su casa para ir juntos al punto de encuentro, dejándome claro que ella

\footnotetext{
educación pública, gratuita y laica, como lo explican en los panfletos que entregaron para el aniversario de la muerte por los siete meses. 13 Partido político de centro izquierda fundado en 2008 por el diputado nacional Carlos Heller.

14 Este sector de la plaza era un espacio con placas recordatorias sobre los desaparecidos durante la última dictadura militar. Un espacio reclamado por Elvira -representante de la organización política "La Mesa de Derechos Humanos de Punilla"- hacia la municipalidad de Capilla del Monte. Todos los jueves lo limpiaba y colocaba objetos en esa parte de la plaza. Las actividades conmemorativas de la última dictadura militar argentina se producían alrededor del Jardín de la Memoria.
} 
iba solo por su hijo.

De este modo, los medios que fueron establecidos para este ritual estuvieron atravesados por cargas emocionales que tuvieron que ver con los conflictos que había al interior del grupo asambleario y la familia, en torno a las tensiones sobre hacer política con el muerto.

Cuando la madre me explicó los motivos del distanciamiento con el grupo, su énfasis estaba puesto en que eran ellos los que llevaban el dolor de la muerte de un hijo. Distanciándose de la lógica partidaria que entendía realizaban algunos miembros con el uso político.

La política aquí aparece como una práctica que termina por exponer los intereses de los actores. Para su mamá, José era nombrado por algunos asambleístas para la utilización de prácticas con intereses políticos, antes que a la búsqueda de justicia.

En esta misma línea, Diego Zenobi (2010) da cuenta de la relación entre familiares y la política dentro del grupo de Cromañón. Los familiares de diversos grupos deciden distanciarse de los políticos y las agrupaciones para no politizar la búsqueda de justicia. La politización, está representada por la inserción de militantes de izquierda y por funcionarios políticos. Bajo esta línea, Alba enuncia que la política partidaria era promovida a espaldas de la familia. Hacer política, por lo tanto, se construía mediante el uso sin el consentimiento de Alba o su familia, del nombre de su hijo y de las acciones a seguir para la búsqueda de justicia. Hay una ausencia de la participación familiar que se vio tanto en los eventos promovidos en Cosquín, como en la construcción del reclamo para el aniversario.

De esta manera, para la figura familiar había una "contaminación de la política" mediante el interés, al utilizar a José bajo provecho. Al incorporarlo a organizaciones políticas para fines propios y a hacerlo a espaldas, sin un consenso previo y sin participación en las decisiones de la Asamblea.

En oposición, la causa familiar era legitimada desde el desinterés, asociado a lo genuino, movilizado por el dolor que llevan, tal como lo muestran las palabras de Alba, al explicarme que el dolor lo llevaban ellos, que él le daba fuerzas para luchar y que asistía a la movilización sólo por su hijo. La politización de la Asamblea entonces aparecía como una contaminación de las emociones que vehiculiza la familia.

En el primer evento, de esta manera, la figuraba familiar vinculada a los sentimientos, junto a las apelaciones en torno a la sangra, el amor y el dolor. Como me explicó Alba, el dolor familiar era aquello que otorga una autoridad que tornan genuinas sus decisiones y formas de intervención (Pita, 2005; Vianna y Farias, 2011; Vecchioli, 2012).

\section{Yo sabía que a José lo mató la policía. A dos años de la muerte}

Año tras año los eventos que se construían con cada aniversario de la muerte permitían develar el momento del duelo que atravesaban los familiares y la importancia que iba tomando el reclamo para ellos. Hacia los dos años de la muerte de José, el escenario distaba de lo que había ocurrido un año atrás. La figura familiar fue crucial en la manera que ocurrió el evento, el grupo asambleario como colectivo tuvo una participación pormenorizada y las disputas previas parecían alejarse.

Las reuniones de la Asamblea en la plaza habían quedado atrás, sólo algunos miembros seguían manteniendo vínculo con la familia. Débora y Valeria provenían de la provincia de Buenos Aires y hacía poco tiempo que vivían en la ciudad de Capilla del Monte. Ambas militaban en la CORREPI ${ }^{15}$, una organización política que denuncia las políticas represivas del estado argentino. Ellas seguían manteniendo un estrecho lazo con la familia luego de que el colectivo asambleario se disipara. Fueron quienes organizaron junto a la mamá de José una nueva movilización en el centro de la ciudad de Capilla del Monte. La continuidad del reclamo seguía instalado en la ausencia de acciones judiciales para esclarecer la muerte ocurrida en la comisaría. El encuentro, una vez más, sucedía en la plaza.

A diferencia del año anterior vinieron personas desde la ciudad de Córdoba como también medios de comunicación. Aquellos que participaron de la Asamblea se hicieron presente. De la familia de José, por primera vez desde su muerte, participaban todos los miembros. Sus padres, hermanas e incluso uno de sus mejores amigos. Eran ellos los que encabezaban la marcha con una pancarta gigante con el nombre de su hijo confeccionada especialmente para ese día.

La manifestación nuevamente inició en el "Jardín de la Memoria", pero esta vez el nombre de José llegó hasta el frente de la comisaría. Los bombos que sonaban al ritmo de los cánticos de "Yo sabía que a José lo mató la policía" se enfrentaron al cordón de efectivos policiales que se armó para proteger el ingreso principal de la institución qque, dos años atrás, fue escenario de una represión de la gendarmería nacional. Para cerrar el evento, se retornó a la plaza y algunos oradores dieron sus discursos. La primera en hacerlo, una vez más, fue Alba. Luego de llegar hasta la comisaría se mostró muy conmovida. Entre lágrimas agradeció a todos por seguir acompañándolos y no dejarlos solos, no iba a parar, hasta que se haga justicia.

Yo no quería marchar hasta la comisaría, tenía miedo de que

15 Coordinadora contra la Represión Policial e Institucional. Ver en: http://www.correpi.org/ 
se arme quilombo y terminen metiendo preso alguno de los chicos. Pero Malena -su hija mayor-, que es más corajuda, me dijo que lo hiciéramos y vayamos hasta la puerta, le demostráramos que no teníamos miedo. Y fue así, pudimos cantarles a la cara que me asesinaron a mi hijo, le pudimos demostrar que no le tenemos miedo y que vamos a estar hasta el final (Alba, octubre 2015).

Luego de que hablara la madre, una de las jóvenes presentes, Débora, repasó las adhesiones de diferentes organizaciones a la marcha. También leyó una carta escrita por Diego, el amigo de José, contando lo mucho que lo extrañaba, caracterizando los detalles de la personalidad de José y cuanto había aprendido a su lado. Mientras la leía lloraba, como así también lo hacía Alba con sus hijas. Cuando finalizó la lectura, levantó la carta con su mano.

Es impresionante la cantidad de pedacitos de alma que se llevan cada que vez que matan a un pibe (Débora, octubre 2014)

Al igual que su amigo, la hermana narró en su carta lo mucho que lo echaba de menos, el dolor que le causó la muerte y la ausencia de su cuerpo: "Te quiero abrazar, pero hay un maldito cajón de madera que nos separa".

La lectura de las cartas que recordaban a José reproducía una comunicación que ponía en relieve una gramática en donde la corporalidad y las emociones, organizaron y experimentaron dicha situación (Daich, Pita y Sirimarco, 2007). Como explica Tambiah (1986 en Peirano, 2002) uno de los valores performativos del ritual radica en la experimentación intensa del evento por parte de los participantes

Este momento emotivo marcado por lágrimas y el dolor de la pérdida finalizó cuando los representantes de las distintas organizaciones sociales presentes fueron desarrollando un pequeño discurso de reclamo. Como el año anterior, estaban el Frente de Izquierda de los Trabajadores, La Asamblea Permanente por los Derechos Humanos (APDH) y el Partido Obrero.

Volvió a tomar la palabra Alba, esta vez me miró y me agradeció por estar allí presente en este nuevo aniversario y que junto a los demás le dábamos fuerzas, porque a veces se caía como toda madre, pero era fuerte $e$ iba a luchar hasta el final, por más que vayan a su casa miles de veces. Esto le permitió contar que el día anterior, un patrullero se paró al frente de su casa para preguntarle si iba a realizar una manifestación para el día de hoy. Bajo el grito de "José presente, ahora y siempre", se dio por finalizada la convocatoria.

Este nuevo escenario en el que se produjo la movilización pudo dar cuenta de los cambios al interior de las relaciones entre los miembros más antiguos de la Asamblea y la participación activa de la familia. Lo que puso en el foco la importancia de la figura familiar como parte del reclamo y el lugar que ocupan sus emociones y el duelo. A diferencia del año anterior, la participación del núcleo familiar estuvo ligado a expresar y reclamar por la pérdida. A través de Fernández Álvarez podemos comprender que las emociones pueden pensarse como prácticas políticas capaces de establecer un modo de comunicación, al mismo tiempo verbal y no verbal, que ponen en juego el registro íntimo en el que intervienen relaciones afectivas y experiencias próximas (2011:18).

Tal como Pita (2010) y Zenobi (2014) explican, los "lazos de sangre" funcionan como impulsores de la intervención y la acción pública. Se plantea el "locus del dolor" como aquello que le otorga legitimidad a la acción política de los familiares, como parte del reclamo de la muerte de sus seres queridos. Las emociones de los familiares son politizadas como parte fundamental del reclamo, transformando lo público en privado, a través del dolor y la bronca (Pita, 2010). De modo tal que los repertorios emotivos forman parte de las acciones políticas habilitadas en el reclamo público.

\section{Se va a acabar esa costumbre de matar. A los tres años}

El correr del tiempo no inhabilitaba el espacio de encuentro que se construía cada 26 de octubre. Una manifestación del reclamo de justicia que seguía sin avances en la investigación. Las actividades de este año habían comenzado días antes con un taller con otros familiares de víctimas de violencia institucional junto a pintadas de murales y grafitis en diversos espacios de la ciudad.

El "Jardín de la Memoria" se constituyó en el espacio de encuentro año a año para iniciar las movilizaciones. Para esta ocasión se hicieron presentes familiares de jóvenes asesinados por la policía cordobesa. Formaban parte de la "Coordinadora de Familiares de Víctimas de Gatillo Fácil"16 y viajaron desde la ciudad de Córdoba para participar de la movilización. De la misma manera que en los años anteriores, asistieron organizaciones políticas como el Frente de Izquierda.

Los miembros de la Coordinadora encabezaron la marcha junto a la familia de José, que una vez más participaron todos sus miembros. Cada madre llevaba una remera

\footnotetext{
16 Esta agrupación se formó en 2015, a partir de los diversos casos de violencia policial que hubo en la ciudad de Córdoba. En el momento en el que se cumplían tres años de la muerte de José, estaba conformado por 6 casos de jóvenes, cuyas madres, tías y hermanas eran las que organizaron este espacio con un solo padre.
} 
estampada con la cara y el nombre de su hijo, mientras sostenían en lo alto carteles reclamando justicia por esas muertes. La consigna de "Basta de torturas y asesinatos en las comisarías" fue la más replicada en las personas que se movilizaron y llevaban carteles alusivos al respecto.

La comisaría fue nuevamente escenario de cánticos y esta vez también de insultos, aunque sólo salieron algunos policías a la puerta mientras se hacían grafitis en la pared que estaba al frente. Allí se pusieron los nombres de los jóvenes muertos que formaban parte de la Coordinadora.

Los insultos con connotación sexual estaban dirigidos especialmente a una de las policías que estuvo el día de la muerte de José y que formaba parte del espontáneo cordón que apostaron en la puerta. Como explica María Pita (2010) los insultos, como así también los cánticos, expresan de manera ritualizada, una relación de poder que se pretende invertir. Se construye un modo de operar en el espacio público que busca expresar, visibilizar y traducir las experiencias en torno a la violencia policial.

Los cánticos, los grafitis y los lugares donde se produjo la marcha fueron organizados por las madres de la Coordinadora. Así se marchó hacía el centro de la ciudad hasta la techada ${ }^{17}$, cuyos comerciantes se habían mostrado en contra de las manifestaciones cuando sucedieron las primeras marchas, los días siguientes de la muerte en 2013.

Desde que inició la marcha, entre aplausos se realizaron cánticos en contra de la policía:

\section{-Se va a acabar esa costumbre de matar}

-Ya van a ver, las balas que vos tiraste van a volver.

La marcha finalizó regresando a la plaza central, donde cada uno de los familiares presentó los casos de violencia policial que los había constituido en activistas por el reclamo de justicia. Entre lágrimas, cada una de las madres, especificó cómo fueron asesinados sus hijos y el estado del proceso judicial en ese momento. Se finalizó cuando se leyó la lista de todos los jóvenes asesinados y en un abrazo entre todas las madres.

El recuerdo de los tres años de la muerte de José sumó nuevos protagonistas que permitieron enfatizar en la fuerza del lazo materno en el accionar de reclamo. Una representación del dolor manifestada a través de las remeras y carteles que llevan cada una de las madres, poniendo en escena cómo lazo primordial del sufrimiento. Se produce una denuncia pública que, a través de las muertes singulares, promueven una pretensión de universalidad para legitimar el reclamo, yendo de lo

17 La techada es una calle cercana a la plaza principal, que está cerrada. Fue construida en 1964 para albergar una exposición fotográfica, convirtiéndose en uno de los atractivos turísticos de Capilla del Monte, donde se encuentran los principales centros comerciales. singular a lo colectivo (Boltanski, 2000).

Esto se constituye a través del lenguaje moral y afectivo de las madres como actores políticos que trazan lo femenino en sus cuerpos (Vianna y Farias, 2011). El foco en el reclamo del tercer aniversario está protagonizadola presencia de las figuras maternas que acompañaron a la mamá de José. Produjeron una universalización aún mayor que las acciones ritualizadas en los años anteriores, construyendo una jerarquía moral materializada en el género y el lazo (Vianna y Farias, 2011).

\section{Entre dramatizaciones y legitimidades}

A través de la descripción etnográfica de los eventos transcurridos intenté hacer un acercamiento de la manera en que esos procesos rituales reflejan los modos de dramatización del reclamo y las relaciones y disputas que se construyen alrededor. Los rituales fueron meros enunciados sobre las maneras que estuvieron atravesadas las emociones, la politización y el lugar de la figura familiar. Siguiendo esta dirección, se pueden enunciar esos tres momentos del siguiente modo:

1)- En ese primer aniversario fue Elvira quien, como activista política, organizó la movilización y promulgó los abrazos entre los asistentes. En el primer año,la política surge como una categoría que deslegitimaba y desactivaba el reclamo por la muerte de José. Las actividades organizadas estuvieron atravesadas por la contaminación de la política que no ponía el acento en la centralidad de la figura familiar y el dolor que ellos tenían por la pérdida.

2)- El dolor por la muerte fue un eje central que configura un aniversario plagado de símbolos que representaban la pérdida y el reclamo, donde el duelo familiar cobróprotagonismo. Las emociones aquí se ponen de relieve para conmemorar un nuevo año y tuvieron una presencia mayor en la movilización pública. Esto es escenificando poniendo el cuerpo en la puerta de la institución donde murió José por primera vez desde que conocieron el suceso. Los abrazos que ocurrieron al finalizar los discursos sucedieron entre la madre y sus hijas.

3) A los tres años, se construye una legitimidad de la figura materna a través de la colectivización del reclamo con otras madres que acompañaron el reclamo, a través de la unificación de los casos particulares. Este último evento pone de relieve la construcción de la figura materna como activista, consagrada a través de la materialización del género y el lazo. Los abrazos sucedieron entre las madres una vez que cada uno narró la muerte de sus hijos.

Las rituales realizados en cada aniversario permitieron reflejar esta construcción de "activistas" por parte de la familia de José, en cuanto a las conductas repetidas durante las puestas en escena pero que se fueron 
resignificando y dándole otro lugar a esos espacios. Los abrazos son la escenificación del lugar que fueron ocupando los familiares, como atravesaron esas experiencias del reclamo público y transcurrieron el dolor. Cada instancia estuvo formada por especificidades que fueron reconstruyendo esa legitimidad, a través de acciones, sujetos, discursos y modos de ocupar el espacio que fueron mutando.

Se trata de un proceso del duelo que no está imbricado sólo en el tiempo que transcurrió, sino también en los actores sociales que fueron formando parte. Una transformación de la madre de José en una activista mediante la colectivización del dolor, que, de la misma forma, se produjo a través de la familiarización con las organizaciones reflejado en ese último abrazo entre las madres. También enraizada en el agradecimiento sobre aquellos que a pesar del tiempo transcurrido siguen acompañando el reclamo, incluyendo a los miembros del colectivo asambleario. Las emociones, los insultos y la circulación en la ciudad, fueron diversificándose en la construcción del lenguaje de la protesta (Alencar Chaves, 2002).

Dar cuenta de estas transformaciones en el reclamo es problematizar y desandar el trabajo político en el que se producen resistencias y tensiones. Por ello me parece necesario retomar a Diego Zenobi (2010) y comprender las nociones desde los significados situados de la política. Es decir, una categoría nativa que está inscripta en las prácticas que los familiares y asambleístas producen, en relación a los contextos y en función de qué fines.

La supuesta polarización entre las concepciones de familia y política que surgen en un primer momento pone de manifiesto las expresiones situadas, cuyos sentidos son disputados por los actores. Las actividades políticas de cada reclamo tienen múltiples sentidos en torno a las acciones que llevan a cabo. De modo tal, que las disputas sobre la legitimidad de las figuras y acciones para reclamar están atravesadas por los procesos políticos que habilitan -o no- esas significaciones.

\section{Bibliografía}

Alencar Chaves, Christine. (2002). "A Marcha Nacional dos Sem-terra: estudo de um ritual político". En: Mariza Peirano (org.), O dito e o feito: ensaios de antropologia dos rituais. Rio de Janeiro, Brasil: Relume Dumará,

Boltanski, Luc. (2000) El Amor y la Justicia como competencias. Tres ensayos de sociología de la acción. Buenos Aires: Amorrortu Editores.

Daich, D; Pita M. V y Sirimarco M. Configuración de territorios y control policial: corporalidades, emociones y relaciones sociales. Cuadernos de Antropología Social N²5 pp71-88, 2007.

Gluckman, M. (2003). Análisis de una situación social en la zululandia moderna. Bricolage 1: 34-49.

Peirano, Mariza.(2002) "A análise antropológica de rituais". En: Mariza Peirano (org.), O dito e o feito: ensaios de antropologia dos rituais. Brasil: Relume Dumará.

Pita, María Victoria (2010). Formas populares de protesta: Violencia policial y familiares de gatillo fácil. En Astor Massetti, Ernesto Villanueva, Marcelo Gómez (comps) Movilizaciones, protestas e identidades políticas en la Argentina del Bicentenario. Bs As: Nueva Trilce.

Schechner, Richard. (2000). Performance. Teoría \& Prácticas Interculturales. Buenos Aires: Libros del Rojas.

Turner, Víctor. (1999). La Selva de los Símbolos. Madrid: Editores Siglo Veintiuno.

Vecchioli, Virginia (2012) "Repertorios militantes y expertise jurídica en la defensa de la causa de los Derechos Humanos en la Argentina" el caso de la Liga Argentina por los Derechos del Hombre. Revista Ensemble Año 6 - nº 9.

Vianna, Adriana y Farias, Juliana. (2011) "A guerra das mães". Dor e política em situações de violencia institucional. São Paulo: Cadernos Pagu.

Viillarreal, Agustín (2016) Al dolor lo llevamos nosotros. Una etnografía en torno a emociones, política y muerte en Capilla del Monte (Córdoba). Tesis de Lic. en Antropología. FFyH- UNC.

Zenobi, Diego (2010) Los familiares de víctimas de Cromañón, en la encrucijada del "dolor". Emociones, relaciones sociales y contextos locales. RBSE. Revista Brasileira de sociología da emocao. Joao Pessoa; Año: 2010 vol. 9.

Zenobi, Diego (2014) Familia, política y emociones. Las víctimas de Cromañon entre el Movimiento y el Estado. Editorial Antropofagia, Ciudad Autónoma de Buenos Aires. 